\title{
Christophe DEJOURS, Florence BÈGUE, Suicide et travail, que faire?
}

Paris, Presses universitaires de France, coll. Souffrance et théorie, 2009, $130 \mathrm{p}$.

\section{Julien Pierre}

\section{(2) OpenEdition}

1 Journals

\section{Édition électronique}

URL : http://journals.openedition.org/questionsdecommunication/313

DOI : 10.4000/questionsdecommunication.313

ISSN : 2259-8901

\section{Éditeur}

Presses universitaires de Lorraine

\section{Édition imprimée}

Date de publication : 1 décembre 2010

ISBN : 978-2-8143-0056-9

ISSN : 1633-5961

\section{Référence électronique}

Julien Pierre, «Christophe deJours, Florence bèGue, Suicide et travail, que faire? 》, Questions de

communication [En ligne], 18 | 2010, mis en ligne le 09 mai 2012, consulté le 22 septembre 2020. URL : http://journals.openedition.org/questionsdecommunication/313; DOI : https://doi.org/10.4000/

questionsdecommunication.313 
Christophe Dejours, Florence BĖGue, Suicide et travail, que faire?

Paris, Presses universitaires de France, coll. Souffrance et théorie, 2009, 130 p.

France Télécom, Renault, EDF, Peugeot: ces grandes entreprises ont - au moins - une caractéristique commune, celle d'avoir donné du grain médiatique à moudre et de relancer le débat sur la souffrance psychique au travail et ses conséquences. Comme le suicide qui, faut-il le rappeler, ne se limite pas aux entreprises précitées: on se défenestre aussi chez les pompiers ou dans les administrations publiques. S'il demeure délicat et malaisé de recenser avec précision les suicides explicitement dus aux conditions de travail (harcèlement, burn-out, etc.), tout porte à croire que le nombre de victimes reste sous-estimé, ceci en dépit de la recrudescence, ces dernières années, des suicides sur le lieu de travail 
(fait jusqu'alors rarissime). Peu importe le nombre finalement, tant un seul de ces drames suffit pour affecter l'ensemble d'une communauté de travail et justifier la nécessaire reconstruction du tissu social interne. Face à l'augmentation - non pas tant celle des suicides au travail mais de leur traitement médiatique - des chercheurs s'attachent à trouver des solutions pour lutter contre le malaise ambiant et la souffrance à l'œuvre dans une majeure partie des entreprises modernes. C'est la mission que se sont fixés Christophe Dejours, professeur au Conservatoire national des arts et métiers (CNAM) et psychanalyste connu pour être l'un des pionniers du traitement de la souffrance (en France), et Florence Bègue, psychologue du travail et consultante en entreprise.

Justement, leur ouvrage s'appuie - au moins pour ce qui concerne sa partie « pratique » - sur une intervention menée par cette dernière dans un site industriel après une vague de suicides. S'lls expliquent d'emblée que l'objet est de rassembler les éléments d'une méthode d'investigation et d'action, il ne faut pas s'attendre à feuilleter un « guide des actions post mortem à mener » (pensons-nous). Pour le dire autrement, il convient de ne pas appréhender cet ouvrage comme un manuel. D'abord parce que les auteurs n'ont pas la prétention de livrer des recettes applicables à l'ensemble des dysfonctionnements observables dans les entreprises du XXle siècle. Ensuite, parce que les sciences humaines ne sont pas une science exacte. L'ouvrage expose, simplement, les conditions de l'intervention d'une consultante (Florence Bègue). II faut dire que les réactions collectives (ou l'absence de réaction collective - parfois, mourir sur son lieu de travail ne suscite aucun émoi) qui suivent un suicide méritent un examen attentif. Ainsi les auteurs s'attèlent-ils à « signaler l'extraordinaire puissance de résistance que les suicides au travail opposent à la recherche de la vérité » (p. I0).

Dans le premier chapitre, Christophe Dejours explique pourquoi et comment toute expression de la souffrance sur le lieu de travail semblait tacitement bannie. Cet ethos professionnel, teinté de virilité, de prise de risque et de mépris contre un habitus efféminé, explique que les gestes suicidaires se déroulaient à distance du lieu de travail, comme sous la pression d'une ultime pudeur, socialement construite. Ensuite, il souligne l'importance - et l'influence - de la solidarité entre collègues. L'effritement des liens sociaux, notamment des forces agrégatives que constituent les syndicats, justifie également cette fragilité sociale. Aujourd'hui, « un collègue s'enfonce, on ne lui tend pas la main » (p. 20) écrit, de manière un brin caricaturale, l'auteur. Ce dernier souligne trois sources qui s'opposent dans l'interprétation des liens entre suicide et travail : le stress constitue une première raison majeure entraînant des perturbations biologiques et psychiques; la seconde attribue toute conduite pathologique (dont le suicide) à des « failles ou à une vulnérabilité individuelles »; la troisième analyse, baptisée 《sociogénétique » », stipule que les contraintes des méthodes de management et l'organisation du travail sont décisives. Comme l'avait remarqué en son temps Marie-France Hirigoyen (Le harcèlement moral, Paris, Éd. La Découverte/Syros, 1998) à propos des victimes de harcèlement moral, le « profil type » du suicidé n'existe pas. Et s'il fallait à tout prix le brosser, l'individu en question serait plutôt impliqué dans son travail, travailleur apprécié de sa hiérarchie et de ses collègues. Tout sauf un « bras cassé » en somme. On découvre alors à quel point la frontière entre vie professionnelle et vie privée est ténue, fragile et que cette coquille se brise rapidement dès lors qu'un salarié se heurte à de lourdes difficultés relationnelles, est mis au placard ou, à l'inverse, croule sous la charge de travail. « Préoccupé, irritable, peu disponible pour ses proches, il [le salarié] gêne la vie des membres de sa famille » (p. 30) :lorsque les soucis professionnels s'invitent dans l'espace domestique, c'est toute la famille qui est appelée à coopérer et « le prix à payer par tous peut devenir exorbitant » (p. 3l). Christophe Dejours (De la psychopathologie à la psychodynamique du travail. Addendum à la Ze édition de «Travail : usure mentale », Paris, Bayard, 1993) met directement en cause trois évolutions majeures introduites récemment dans l'organisation du travail. Le privilège accordé à la gestion aux dépens du travail a permis l'introduction de nouveaux instruments de contrôle et a contribué à atténuer la référence au travail bien fait. Associée à une évanescence de différentes formes de reconnaissances symboliques, ces évolutions ont un impact majeur sur l'identité professionnelle et le bien-être au travail des salariés. Autre évolution importante: l'évaluation individualisée des performances. Cette méthode, qui repose sur des «bases scientifiques erronées 》, génère des concurrences internes et fissure les solidarités. Enfin, la « qualité totale » réclamée par les dirigeants, doublée d'injonctions paradoxales (entre autonomie et contrôle), provoque une souffrance non négligeable.

Le deuxième chapitre de l'ouvrage plonge dans les ateliers de Mermot. On suit les pérégrinations de Florence Bègue. Après avoir minutieusement dépeint le contexte spatio-temporel de son intervention, la consultante expose les étapes de son 
intégration progressive et de la réflexion collective qu'elle a impulsée dans l'usine. Tout commence par une rencontre avec les dirigeants, puis par une participation au comité d'hygiène, de sécurité et des conditions de travail (CHSCT) et par une mise en place d'un comité de pilotage. Elle explique pourquoi elle a souhaité obtenir un bureau excentré, à l'abri des regards des collègues, relatant quelques confidences de ceux qui s'y sont arrêtés. Elle raconte ensuite son voyage au cœur même des ateliers, expliquant le bienfondé de cette immersion pour saisir le contraste avec les entretiens individuels. Puis vient le temps de la rédaction d'un document de synthèse, diffusé à l'ensemble du personnel, dressant les principaux enseignements liés à l'intervention de la consultante. Un rapport qui servira de base à des débats internes sur l'organisation du travail. Grâce à ces dispositifs, Florence Bègue met au jour différents dysfonctionnements - que l'on découvrira au fil de la lecture - et délivre quelques recommandations ciblées notamment sur un nécessaire renforcement de la coopération horizontale, mais aussi verticale.

L'ouvrage se clôt sur 20 pages de commentaires méthodologiques où Christophe Dejours s'efforce de tirer quelques « leçons» générales de l'expérience des ateliers de Mermot. Solidité des références théoriques, indépendance et discrétion du praticien, primat du travail (objectif) sur la commande (subjective), formation d'une équipe d'intervention, entretiens individuels, etc.: l'auteur passe en revue les différentes balises par lesquels tout consultant qui se respecte devrait passer s'il recevait une demande similaire. Ce dernier développement contribue à faire de cet ouvrage, écrit à quatre mains qui ne se croisent jamais, un exemple - voire un « modèle », comme le précisent les auteurs - précieux pour qui souhaite infléchir un climat social dégradé au sein d'une organisation.

Julien Pierre

ERSSS, HMS, université de Strasbourg julien.pierre@unistra.fr 